\title{
Physical aggression and attentional bias to angry faces: An event related potential
} study

Rebecca V. Crago, Louis Renoult*, Laura Biggart, Gavin Nobes, Tamara Satmarean ${ }^{\text {a }}$, Jennifer O. Bowler

Corresponding author: Louis Renoult Email: 1.renoult@uea.ac.uk*

School of Psychology, Lawrence Stenhouse Building (LSB), University of East Anglia, Norwich Research Park, Norwich, NR4 7TJ, United Kingdom.

$$
\begin{gathered}
\text { r.crago@uea.ac.uk } \\
\text { 1.renoult@uea.ac.uk } \\
\text { 1.biggart@uea.ac.uk } \\
\text { g.nobes@uea.ac.uk } \\
\text { tssatmarean1@sheffield.ac.uk } \\
\text { j.bowler@uea.ac.uk }
\end{gathered}
$$

aPresent address: Department of Psychology, The University of Sheffield, Cathedral Court, 1 Vicar Lane, Sheffield, S1 2LT, United Kingdom. 


\begin{abstract}
This study aimed to identify the neural correlates of aggression-related attentional selectivity to angry faces in physical aggression. Physical aggression in a non-clinical sample of young men $(N=36)$ was measured using an aggression questionnaire. Visual attentional bias to angry faces was assessed using a dot-probe task during which angry and neutral faces were presented simultaneously, and EEG was recorded. Median split and correlational analyses were conducted to assess the relationship between physical aggression and attentional bias.

Behavioural results indicated that higher levels of physical aggression were associated with greater attentional bias to angry faces. ERP results revealed an interaction where males with higher physical aggression had undifferentiated P3 amplitudes to angry and neutral trials, whereas low physical aggression males exhibited greater P3 amplitude to angry than to neutral trials (effect of probe congruency). Increased levels of physical aggression were also significantly correlated with increased P3 amplitude to probes replacing neutral faces, relative to angry faces. It was concluded that the aggressive males selectively attend to angry faces, and that attentional bias is characterized by undifferentiated P3 amplitude. We propose that this results from an inferior ability to downregulate competing angry face distractors when responding to probes replacing neutral faces (as reflected by the $\mathrm{P} 3$ response). These findings indicate that attentional bias to angry faces in individuals with higher physical aggression is characterized by a distinctive ERP signature; this could inform the development of therapeutic interventions seeking to reduce aggression.
\end{abstract}

Keywords: physical aggression; attentional bias; angry faces; event-related potential; P3 
Highlights:

- Physical aggressors' attention is biased to angry faces, characterized by a distinct ERP signature.

- More aggressive people show similar P3 amplitudes to angry and neutral faces.

- Their P3 amplitude response to neutral faces suggests poor inhibition of angry face distractors. 


\section{Introduction}

Cognitive theories of aggression (e.g., Crick \& Dodge, 1994; Wilkowski \& Robinson, 2010) highlight the role of increased attention to aggressive cues over non-aggressive cues (attentional bias; e.g., Mellentin, Dervisevic, Stenager, Pilegaard \& Kirk, 2015 for review). According to these theories, hostility-related selective attention, which drives aggression, is the product of increased stimulus-driven attentional capture by angry cues, combined with suboptimal effortful regulatory control (e.g., Strack \& Deutsch, 2004; Wilkowski \& Robinson, 2010). Biased selectivity in aggression is particularly associated with later stages of attention when ruminative processes and difficulties in disengaging from hostile stimuli can influence attentional capture (Wilkowski \& Robinson, 2010). Nevertheless, attentional bias is not based on conscious awareness of the stimuli and is thought to occur at a relatively automatic level of processing (e.g., Beck \& Clark, 1997; Mogg et al., 1993, 1995b; 1998; Wiers, Gladwin, Hofmann, Salemink, \& Ridderinkhof, 2013). Numerous studies have demonstrated visual attentional bias towards negative stimuli in psychopathology. For example, in anxiety attentional bias is indicated by impaired processing of target benign words or images in the presence of threat distractors (e.g., Bar-Haim et al., 2007 for review). It is likely that different disorders are characterized by different processing biases (Beck \& Clark, 1997; Wilkowski \& Robinson, 2010), yet comparatively few studies have investigated attentional bias in aggression.

Behavioural paradigms used to examine valence-driven attentional allocation in aggression have included the dot-probe task (e.g., Maoz et al., 2017; Smith \& Waterman, 2003) and the emotional Stroop task (e.g., Brugman et al., 2016; Smith \& Waterman, 2003). The dotprobe task (MacLeod, Tata, and Mathews, 1986; MacLeod et al., 2002) was designed to assess the relative allocation of attention to simultaneously presented aversive and neutral stimuli, thereby providing a more direct assessment of competition models of attentional selectivity (e.g., Desimone \& Duncan, 1995) compared with tasks that present stimuli centrally one at a time (Bishop 2008; MacLeod, Mathews, Tata, 1986). For example, Smith and Waterman (2003) 
presented aggression-related (violent) and neutral word pairs onscreen for 500ms to violent offenders, non-violent offenders, and an undergraduate control group. Violent offenders responded significantly more rapidly to probes replacing violent versus neutral words in comparison with the undergraduate control group, suggesting that the violent offenders group attended more rapidly to that region of the visual display. However, reaction time measures used in these studies represent not only the cognitive processes of interest, such as selective attention, but also a complex combination including evaluative, decision, and motor processes. To understand the cognitive processes involved with hostile-related attentional bias in individuals with differing levels of aggression, the use of neurological research methods may prove beneficial.

The event-related potential (ERP) technique provides a direct measure of neural activity evoked by events of interest and allows partial isolation of distinct cognitive processing stages (reviewed in Luck, 2005). The P3 waveform comprises several distinguishable ERP components and the term is most often used in the literature to refer to the P3b component. The latter is only present for task-relevant stimuli, in other words, for stimuli which are expected to some degree in the context of the task being completed (for review see Polich, 2007). This ERP component appears as a positive deflection at posterior parietal sites between $300 \mathrm{~ms}$ and $800 \mathrm{~ms}$ after stimulus onset. It is particularly sensitive to selective attention, that is, the differential processing of stimuli in relation to their task relevance (Coles, Smid, Scheffers, \& Otten, 1995; Polich, 2007). The temporo-parietal attentional network appears to be a crucial generator of this ERP component (Knight et al., 1989; Verleger et al., 1994; Yamaguchi \& Knight, 1992).

There is growing evidence from the anxiety literature that attentional bias towards threat stimuli (mood-congruence) could be associated with variations in the amplitude of the P3 during the dot-probe task (Leutgeb, Sarlo, Schöngassner, \& Schienle, 2015; Sass, Evans, Xiong, Mirghassemi, \& Tran, 2017), providing an electrophysiological marker of distorted attentional allocation to threat (MacNamara, Kappenman, Black, Bress, \& Hajcak, 2013). In aggression, 
attenuated P3 amplitude has been associated with adult antisocial behaviour (Gao et al. 2013) and violent offending (Bernat et al., 2007), which may reflect ineffectual processing of salient social stimuli and compromised executive function and response inhibition (Bernat et al., 2007; Patrick, 2008). Although visual attentional bias measured using the dot-probe task is also associated with physically aggressive behaviour (Smith \& Waterman, 2003), no studies have measured the ERP correlates of dot-probe attentional bias in physical aggression, and none have investigated the neural correlates of attentional bias to angry faces in this population.

Nevertheless, a handful of studies have investigated ERP and valence-driven attentional selectivity in aggression using the modified oddball task (e.g., Helfrizt-Sinville \& Stanford, 2015; Surguy \& Bond, 2006). During this task, aversive and benign stimuli are presented individually on screen at different pre-determined frequencies. Two types of neutral word occur with a set frequency (e.g., 20:80): rare neutral stimuli (target) and frequent neutral stimuli (non-target). Also presented are non-target valenced stimuli at the same frequency as the neutral rare stimuli. The participants' task is to respond to the rare neutral target words and ignore the non-target (neutral and valenced) words. These studies report evidence of less differentiated P3 activity between threat-related and neutral words in individuals with high trait aggression, which may reflect similar allocation of attentional resources to process both types of stimuli (Helfritz \& Stanford, 2015). In their study, Helfritz-Sinville and Stanford (2015) used a modified oddball task to assess the P3 component in relation to attentional biases in the processing of threat words. They investigated how two major subtypes of physically aggressive men - reactive (impulsive) and premeditated - process social and physical threat words compared with non-aggressive individuals. They found that the non-aggressive group showed increased P3 amplitude to both social and physical threat words compared with neutral words. This enhanced processing was not demonstrated in the aggressive groups. Reactive and premeditated aggressors had similar P3 response to threat words and neutral words. 
Collectively, findings suggest a behavioural bias towards aggression-related words in line with current cognitive models of aggression (Wilkowski \& Robinson, 2010). They also suggest that a key electrophysiological marker of selective attention, the P3 ERP component, may be similar across stimulus types in aggression-prone individuals when presented with hostilityrelated and neutral words. However, findings thus far do not demonstrate whether high aggression promotes increased $\mathrm{P} 3$ in response to neutral stimuli, or reduced P3 in response to threat/anger-related stimuli. Moreover, to our knowledge, no studies have used the dot-probe assessment of attentional bias to measure P3 amplitude in response to simultaneously presented angry versus neutral faces.

This work was driven by three main hypotheses. The initial aim was to replicate and extend previous findings through examining whether non-clinical individuals with high trait physical aggression display a visual attentional bias towards angry faces using the dot-probe task. The first hypothesis was that increased physical aggression scores would be associated with increased attentional bias to angry faces.

Second, we aimed to determine the neural characteristics of this bias by simultaneously examining its ERP correlates. The current study investigated neural processing relating to attentional bias, specifically in physical aggression. Compared with anger or hostility, which are implicit forms of aggression, and verbal aggression, which is less severe, physical aggression is a measurable explicit behavioural response which is an expression of anger. We recruited a maleonly sample because males show higher levels of physical aggression than females (Archer, 2004). Inclusion of these variables allowed for comparison with previous work by HelfritzSinville and Stanford (2015), which explored the processing of threat words in impulsive and premeditated physically aggressive men.

Importantly, as the P3 component is often widely distributed over posterior parietal sites (Coles, Smid, Scheffers, \& Otten, 1995; Polich, 2007), we explored between-group differences at parietal electrodes 300 to $500 \mathrm{~ms}$ post-probe onset, which is the typical latency of P 3 effects in 
young adults in signal detection tasks (van Dinteren, Arns, Jongsma, \& Kessels, 2014). This

allowed us to overcome limitations of previous studies that typically reported only a few midline electrodes, and to identify more precisely the neurocognitive processes related to physical aggression. Moreover, to better understand the association between P3 and aggression, we used a novel paradigm to investigate separate ERPs evoked by neutral and angry faces in relation to physical aggression scores. Our second prediction was that, relative to participants with low levels of physical aggression, attentional bias in participants with increased levels of physical aggression would be characterized by similarity in evoked P3 amplitude to probes replacing angry and neutral faces (i.e., no effect of probe congruency).

Finally, it was hypothesised that physical aggression scores would correlate with increased P3 amplitude to probes replacing neutral relative to angry faces.

\section{Results}

\subsection{Group characteristics}

Participants were categorized into high and low aggression groups based on a median split of the Buss and Perry Aggression Questionnaire (BPAQ; Buss \& Perry, 1992) physical aggression subscale score $(M d n=18.0$, range $=9-40)$. The high aggression $(M=27.81, S D=$ 5.81) and low aggression $(M=13.01, S D=2.67)$ groups differed significantly in physical aggression score, $t(29)=9.01, p<.001, d=3.27$. The physical aggression score of the high aggression group was comparable to mean physical aggression scores in violent offenders $(M=$ 27.27, $S D=8.31)$ reported by Smith and Waterman (2003).

\subsection{Behavioural results: dot-probe task. Congruency and Physical Aggression}

Pearson correlations revealed that attentional bias index to angry faces negatively correlated with physical aggression, $r(31)=-.348, p=.047$, indicating that those participants with higher levels of physical aggression responded more quickly when probes replaced angry rather 
than neutral faces (congruent). However, one-way ANOVA results revealed a non-significant effect of congruency, $F(1,29)=2.82, p=.104, \eta_{\mathrm{p}}{ }^{2}=.088$, and congruency did not interact with physical aggression, $F(1,29)=0.93, p=.342, \eta_{\mathrm{p}}{ }^{2}=.031$, although means were in expected directions (see Table 1).

Table 1. Mean reaction times (and $S D$ s) to angry and neutral faces for high and low physical aggression groups

\begin{tabular}{lcccc}
\hline & $\begin{array}{c}\text { High aggression } \\
(n=16)\end{array}$ & $\begin{array}{c}\text { Low aggression } \\
(n=15)\end{array}$ & $\begin{array}{c}\text { Whole sample } \\
(N=31)\end{array}$ & $p$-value \\
\hline Angry faces & $575.72(67.99)$ & $576.30(56.30)$ & $576.97(63.75)$ & .980 \\
Neutral faces & $584.00(65.21)$ & $578.53(60.39)$ & $581.48(63.91)$ & .811 \\
Bias index & $-8.28(18.49)$ & $-2.23(16.24)$ & $-4.52(17.19)$ & .342 \\
$p$-value & .093 & .603 & .141 & $/$ \\
\hline
\end{tabular}

\subsection{ERP Results}

\subsubsection{Interactions between Congruency and Physical Aggression.}

A mixed model ANOVA with congruency (probe replaced angry versus neutral face), electrode (4 levels), and hemisphere (left versus right) as the within-subjects factors, and physical aggression as the between-subjects factor, was conducted on the $800-1000 \mathrm{~ms}$ data (corresponding to $300-500 \mathrm{~ms}$ post arrow probe onset). Results revealed a significant interaction between hemisphere, congruency and physical aggression, $F(1,29)=8.46, p=.007, \eta_{\mathrm{p}}{ }^{2}=0.226$. Further analyses revealed a significant congruency by physical aggression interaction in the right hemisphere, $F(1,29)=7.62, p=.01, \eta_{\mathrm{p}}^{2}=0.208$, which was not qualified by an interaction with electrode, $F(3,87)=0.45, p=.61, \eta_{\mathrm{p}}{ }^{2}=0.015$. There were no significant interactions with physical aggression in the left hemisphere (congruency by physical aggression, $p=.491$; 
electrode by physical aggression, $p=.709$; congruency by electrode by physical aggression, $p=$ $.658)$.

Next, as there was no significant interaction between electrode and physical aggression at either step, amplitudes were averaged across the four right hemisphere electrodes for each trial type (probe replaced angry face / probe replaced neutral face), and paired samples $t$-tests (2tailed) were performed on these data within each physical aggression group. In the higher physical aggression group, mean amplitudes to probes replacing angry faces $(M=3.96, S D=$ 3.62) did not reliably differ from amplitudes to probes replacing neutral faces $(M=4.74, S D=$ 4.25), $t(15)=-1.50, p=.155, d=0.367$ (see Figure 1). In the low physical aggression group, mean amplitudes to probes replacing angry faces $(M=3.81, S D=2.97)$ were significantly higher than to probes replacing neutral faces $(M=2.30, S D=2.80), t(14)=2.32, p=.036, d=0.60$ (Figure 1).

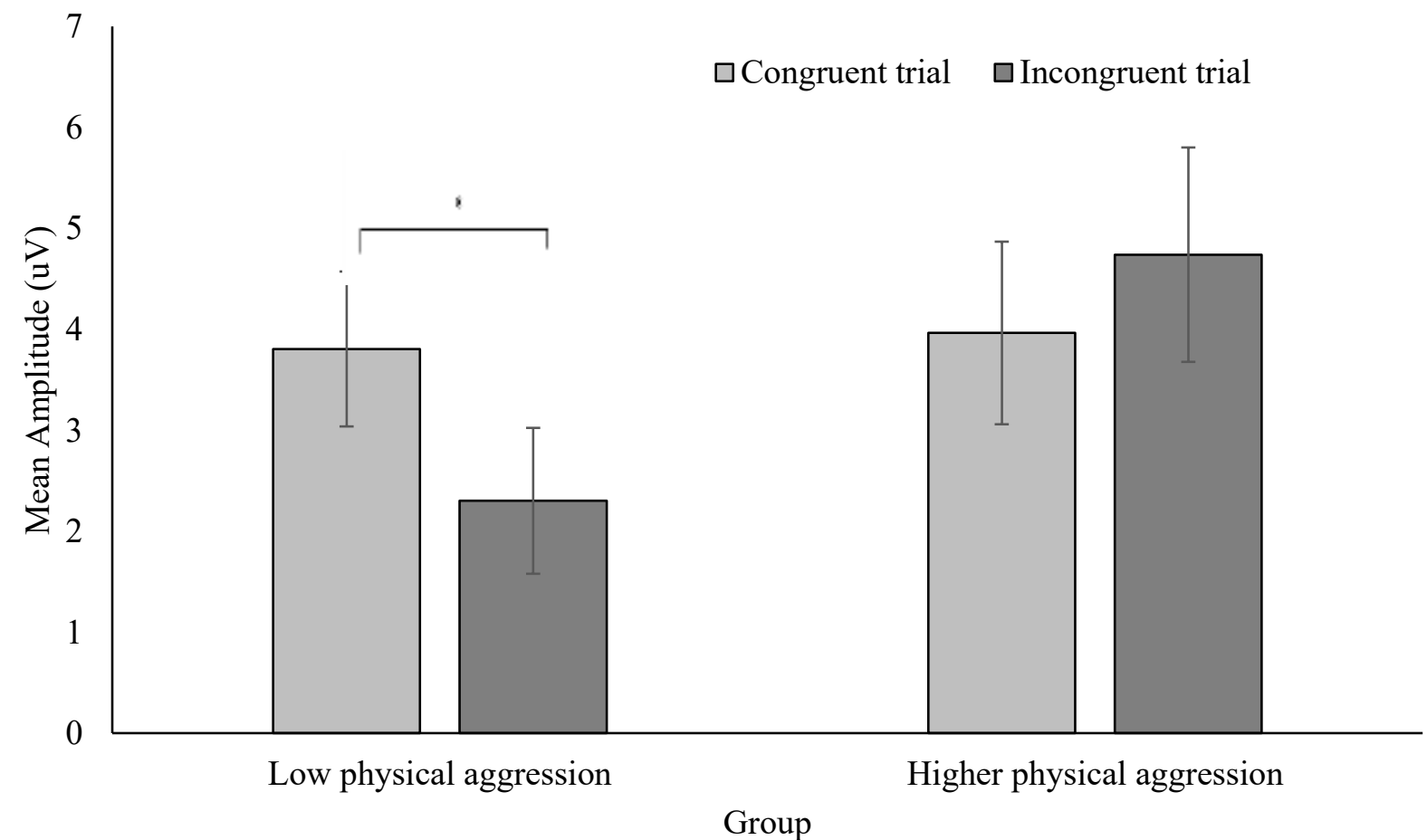


Figure 1. Mean evoked amplitudes (300-500ms post-probe onset) to arrows replacing angry faces (congruent trial) and arrows replacing neutral faces (incongruent trial) during the dot-probe task by physical aggression group. Error bars represent \pm 1 standard error.

Inspection of the waveforms confirmed that angry trials (congruent) were associated with more positive amplitude compared with neutral (incongruent) trials in the low aggression group (see Figure 2). This effect had a classic right-side posterior distribution (e.g., Davidson, 1993; Mogg \& Bradley, 2002; Polich, 2007), with maximal amplitudes distributed across right-sided parietal electrodes, extending to temporo-parietal sites (see Figure 3). Overall, these results suggest that P3 effects of congruency are more salient in the low physical aggression group compared to the high physical aggression group.
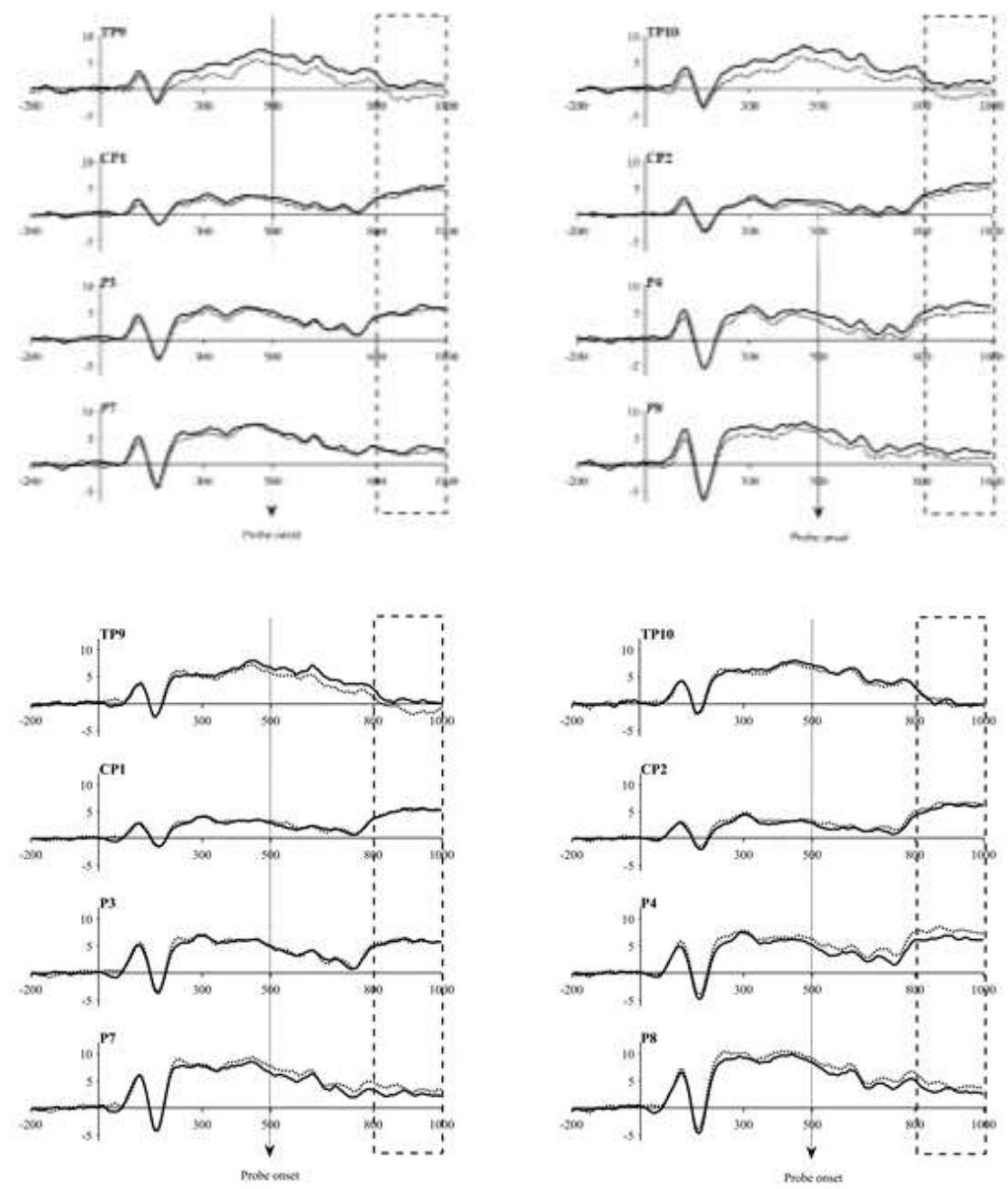
Figure 2. Grand average ERPs for the effect of congruency in participants with low (upper pane; $n=15$ ) and higher (lower pane; $n=16$ ) physical aggression scores. Mean amplitude to congruent trials (black) are compared with mean amplitude to incongruent trials (dotted) for the $300-500 \mathrm{~ms}$ post-probe time window (boxed area).
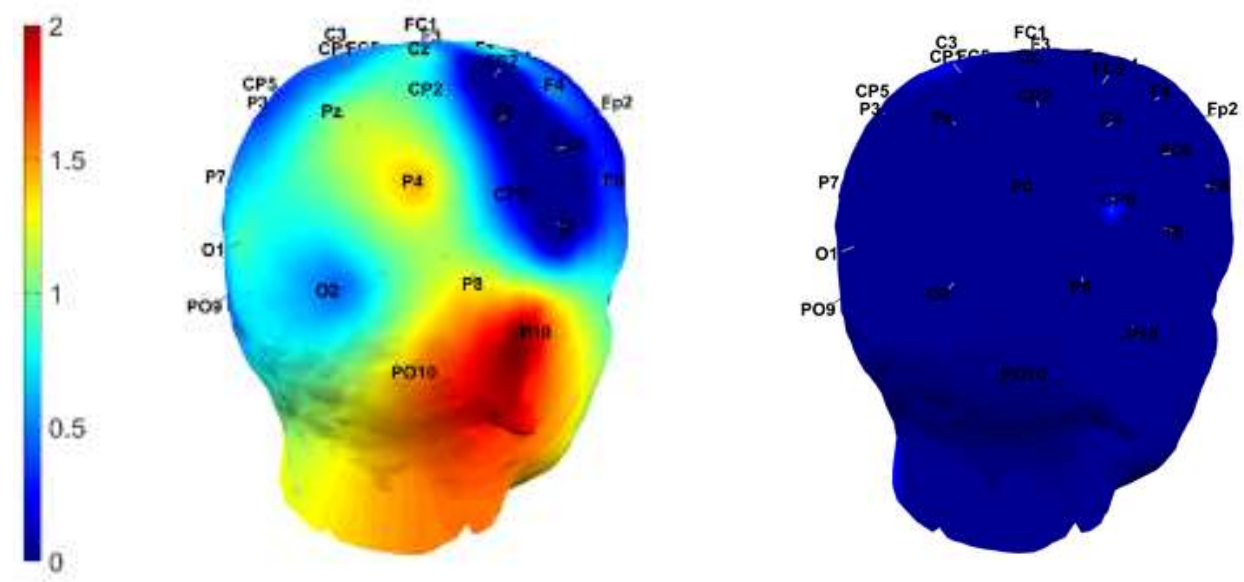

Figure 3. Scalp maps of congruency effects (angry minus neutral) between $300 \mathrm{~ms}$ and $500 \mathrm{~ms}$ post-probe in the low (left pane; $n=15$ ) and higher (right pane; $n=16$ ) physical aggression groups.

Finally, early effects of congruency were also observed before probe onset (300-500ms post-face onset; see Figure 2), confirming that a pre-probe baseline would not have been appropriate (Mingtian et al., 2011; Poulsen et al., 2005), as 300-500 post-face onset would correspond to -200 to 0 pre-probe baseline. An ANOVA indeed showed a near-significant interaction between trial congruency and physical aggression, $F(1,29)=3.26, p=.089, \eta_{\mathrm{p}}{ }^{2}=$ 0.101. The congruency by hemisphere by physical aggression interaction was not significant before probe onset, $F(1,29)=1.14, p=.295, \eta_{\mathrm{p}}{ }^{2}=.038$, and none of the other interactions with physical aggression reached significance (all $F s<1$, except hemisphere by physical aggression, $\left.F(1,29)=3.096, p=.089, \eta_{\mathrm{p}}{ }^{2}=.155\right)$. To further investigate whether congruency effects were also evident before probe onset, the trend-level congruency by physical aggression interaction 
was followed up with paired-sample $t$-tests (2-tailed); these were performed within each physical aggression group on amplitudes averaged across the four electrodes, across both hemispheres. In the higher physical aggression group, there was no difference in amplitude to face-pairs on trials where the probe would replace the angry face $(M=6.09, S D=2.87)$ versus pre angerincongruent trials $(M=6.26, S D=3.22), t(15)=-.365, p=.720, d=-0.09$. In the low physical aggression group, amplitude was unexpectedly higher on average to face-pairs during pre-angercongruent trials $(M=5.37, S D=2.84)$ than during pre-anger-incongruent trials $(M=4.23, S D=$ 3.89), although this difference did not reach significance, $t(14)=2.021, p=.063, d=0.59$.

\subsubsection{Correlation.}

Congruency effects for amplitude were calculated by subtracting the amplitude to probes replacing neutral faces from the amplitude to probes replacing angry faces (Pourtois et al., 2004). A higher positive value therefore represents greater amplitude to angry relative to neutral trials. Pearson's correlation was conducted between physical aggression and this computed P3 index between $800 \mathrm{~ms}$ and $1000 \mathrm{~ms}$ post-face onset (corresponding to $300 \mathrm{~ms}$ and $500 \mathrm{~ms}$ post arrow onset) for the averaged posterior electrodes sites (where effects of trial type and interactions with aggression scores were observed). There was a significant negative correlation between physical aggression and the amplitude bias index, $r(31)=-.404, p=.020$. This suggests that as physical aggression scores increased, P3 amplitude to neutral trials relative to angry trials increased.

Figure 3 also shows larger differences in amplitude across posterior electrodes between congruent and incongruent trials in the low, compared with higher, aggression group. 


\section{Discussion}

\subsection{Overview of results}

This study investigated the relationship between physical aggression and selective attentional processes to angry and neutral faces using both behavioural (reaction time) and ERP measurements. We hypothesized that individuals reporting high levels of physical aggression would have an increased attentional bias to angry faces (larger effect of congruency), and that this bias would be characterized by relatively undifferentiated P3 amplitude across both trial types. Contrary to expectations, there were no between-group differences in reaction time based on dotprobe congruency. In line with our first hypothesis, however, the correlational behavioural results suggested that males with elevated physical aggression had visual attentional selectivity for angry faces (indicated by shorter reaction time to probes replacing angry faces). These findings are consistent with prior dot-probe assessments of attentional bias to hostile words in physical aggression (e.g., Smith \& Waterman, 2003), and extends them to angry faces.

Attentional bias effects were confirmed by ERP data. Consistent with our second hypothesis, congruency effects were found in the low physical aggression group but not in the high physical aggression group. Participants who scored high on physical aggression showed similar amplitudes across angry and neutral trials, whereas participants who scored low on physical aggression showed enhanced P3 amplitude over right electrode sites when responding to angry trials compared to neutral trials, in keeping with studies showing right hemispheric dominance in target-detection tasks (e.g., Mogg \& Bradley, 2002; Polich, 2007; Shulman et al., 2010). This is in also line with findings obtained by Helfritz-Sinville and Stanford (2015), using a modified oddball task with emotional words. They reported similar P3 amplitude across threat (physical and social) and neutral words in aggressive individuals, whereas control participants exhibited enhanced amplitude to the threat words compared to neutral words.

The undifferentiated P3 amplitudes in response to angry and neutral trials in participants with high physical aggression suggest that similar cognitive processing resources are used to 
attend to both angry and neutral faces. A recent systematic review by Mellentin, Dervisevic, Stenager, Pilegaard, and Kirk (2015) proposed that increased aggression is linked to a tendency to perceive hostility in both ambiguous and non-ambiguous non-hostile facial expressions.

Therefore, a possible explanation of the similarity in P3 amplitude across angry and neutral faces in the high physical aggression participants is that they process the neutral faces as if they are hostile. Indeed, greater amygdala and P3 responses have each been noted to neutral faces as well as to angry faces in individuals with impulsive aggression and provoked aggression, in comparison with healthy controls (Coccaro et al., 2007 and Bertsch et al., 2009, respectively). Current data suggest that as physical aggression scores increase, participants display increasing P3 amplitude to probes replacing the neutral faces; it is possible that differences in the processing of neutral faces also contribute to the slower reaction times to the neutral probes. More research is needed on the relative allocation of attentional resources to competing angry and neutral stimuli in aggression to better understand these processes. The behavioural results, and previous findings by Smith and Waterman (2003) and Maoz et al. (2017), suggest that aggressive males differentiate to some extent between aggressive and neutral faces, that is, they have visual attentional selectivity for angry faces indicated by faster reaction times to probes replacing angry faces compared with probes replacing neutral faces. We therefore propose that other explanations, such as the impaired disengagement hypothesis (e.g., Wilkowski \& Robinson, 2010), should also be considered.

A correlational analysis was used to investigate the relationship between physical aggression and selective attentional processes associated with attending to probes replacing neutral and angry faces. Concerning our third hypothesis, results revealed that increased levels of physical aggression were related to increased P3 amplitude to anger-incongruent, relative to congruent, trials in the right parietal region of interest (corresponding to averaged electrode sites $\mathrm{CP} 2, \mathrm{TP} 10, \mathrm{P} 4, \mathrm{P} 8)$. This finding suggests that amplitude to probes replacing neutral faces, relative to when they replaced angry faces, increased with physical aggression in a normative 
sample, possibly reflecting ineffectual downregulation of the angry stimuli. It is proposed that relative uniformity in ERP amplitudes across stimulus types in the higher aggression group could in part be attributed to the recruitment of enhanced cognitive processes on neutral trials (reflected in increased P3 amplitude) to inhibit the response to the simultaneously presented angry face distractor, consistent with an inhibitory account of P3 (Polich, 2007). This pattern of results is in keeping with neurocognitive models of aggression that suggest deficits in regulatory control over incoming perceptual stimuli contribute to visual attentional bias (e.g., Wilkowski \& Robinson, 2010) in physical aggression, with physically aggressive behaviour being characterized by poor emotion regulation and response inhibition (e.g., Patrick, 2008). Theoretically, neural abnormalities in face processing could affect perceptual, cognitive and emotional integration of social cues and contribute to an aggressive response.

The dot-probe task is a paradigm used to capture both facilitative and disengagement biases (Koster et al., 2004), without distinguishing these two processes (see below). A possible explanation of the delayed reaction time and increased positive amplitude to incongruent relative to congruent trials during the dot-probe task is that males with higher physical aggression are less able to disengage from angry stimuli. Therefore, we suggest that disengagement bias may be particularly important for understanding attentional selectivity in aggression. This research provides initial evidence of the ERP correlates associated with selective attentional bias in physical aggression and provides a possible interpretation regarding the complex cognitive mechanisms that underpin the bias. Reaction time data did not provide clear behavioural evidence of a between-groups attentional bias to the angry faces (the congruency by physical aggression group interaction was not significant); one reason for this could be that the dot-probe task in its current form does not distinguish reaction time indices of engagement and disengagement (Clarke, MacLeod, \& Guastella, 2013). Future research could consider using a task that clearly disambiguates these processes in the behavioural data (such as the spatial cueing task; Posner, 1980) to assess whether attentional bias in physical aggression is characterized more by impaired 
disengagement from aggression-related stimuli (as suggested by slower reaction times to angerincongruent probes), than by speeded orienting to these stimuli (faster reaction times to angercongruent probes).

Early effects of congruency were also observed before probe onset, confirming results from other studies using the dot-probe (Mingtian et al., 2011) or similar tasks (Poulsen et al., 2005). Although in the present study, the interaction between congruency and physical aggression was only a trend during this period, there was numerically a near significant P3 effect of congruency to face-pairs in the low physical aggression group for pre-anger-congruent trials, as compared to pre-anger-incongruent trials. Therefore, to avoid any potential contamination of prearrow effects, we chose to use a pre-face onset ERP baseline (similar to Mingtian et al., 2005). These pre-arrow effects are difficult to explain. Because there was no contingency between emotional face position and where the probe would appear, the probe randomly replaced the neutral face for fifty percent of the trials and the angry face for the other fifty percent. Moreover, in our version of the task, combinations of probe type (left or right facing arrow), face type (angry or neutral) and position on screen (top or bottom) were all counterbalanced, with a new random order for each participant. Nonetheless, affect-biased attention to the emotional face of the pair during the face-pair display is suggested by faster reaction times to the probe when it replaced the emotional face (here, the anger-congruent trial), as compared to when the probe replaced the neutral face (the anger-incongruent trial). The assumption is that response will be faster to probes replacing angry faces if attention was in that region of the visual display. In contrast, a more even monitoring of the face-pair display in the normal population is suggested by more similar reaction times to the probe when it subsequently replaces either the angry or neutral face with equal probability. It is possible that during the initial image-pair phase of the test, a more evenly distributed attention in the low physical aggression group was taking place across trials in the region in which the arrow then appeared. In contrast, in individuals with higher aggression, biased visual attention to the angry faces across trials would have resulted in similar 
P3 amplitudes to the later occurring arrow probe for later anger-congruent and anger-incongruent probe-locations (each being equally likely to occur). These pre-arrow trends require further investigation, for instance by using eye tracking to measure whether individuals with higher aggression spend more time looking at the angry faces and are slower to disengage from them. This would help give a more fine-grained analysis of time course.

The current findings have important therapeutic implications. For example, attentional bias is considered a valid therapeutic target across a range of disorders including aggression (Brugman et al., 2016) and anxiety (e.g., Bar-Haim, 2010), and can be targeted using explicit (cognitive behavioural therapy; e.g., Dehghani, Sharpe, \& Nicholas, 2003; Mogg, Bradley, Millar, \& White, 1995a) and implicit (cognitive bias modification (CBM); Bar-Haim 2010 for review) techniques. Attentional bias modification (ABM), which uses computer-based techniques to implicitly modify threat-related attentional bias, has yielded highly successful results in clinically anxious populations (Bar-Haim, 2010; Mogoaşe, David, \& Koster, 2014). Present ERP results suggest not only that EEG is an effective method in measuring attentional processes in physical aggression and therefore could be used alongside current CBM techniques, but also that P3 could be an index used to measure the success of interventions.

\subsection{Limitations}

Behavioural analyses utilizing a between-subjects design based on a median split of physical aggression score failed to provide clear differences in bias indices between groups. However, ERP analyses revealed significant between-subjects effects with medium to large effect sizes. Furthermore, effect sizes reported here were larger than those stated in a previous study by Helfritz-Sinville and Stanford $(2015)\left(\eta_{\mathrm{p}}{ }^{2}=.089\right)$. Moreover, as reported in the methods section, participants of the present study also completed a second dot-probe task in which the face stimuli were replaced with threat-related and neutral words. The main effect reported here was replicated, such that low aggression participants showed significant differences in P3 amplitude 
between angry and neutral stimuli, whereas higher aggression participants showed undifferentiated amplitude across stimulus types (in preparation). Nonetheless, it is recognized that the sample size of this study is relatively small and consequently behavioural analyses that adopted a between-subjects approach are possibly underpowered. Future work using a larger sample size and more extreme groups (very high aggression versus very low aggression) will be needed to replicate these findings and detect significant between-subjects effects for behavioural analyses.

Since this study is correlational and quasi-experimental in nature it is not possible to determine whether attentional bias is a cause or consequence of aggressive behaviour. Also, since the sample was male-only, this study has limited generalizability; further work is required to investigate neural correlates of attention bias in female aggression.

\subsection{Future research}

Within aggressive samples, neutral facial expressions can be perceived as hostile (Mellentin, Dervisevic, Stenager, Pilegaard, \& Kirk, 2015) and therefore neutral faces may not be an optimal control stimulus for assessing attentional bias in aggression. Future research could explore selective attentional processes involved when attending to angry faces paired with other stimulus types, such as happy, sad, or frightened faces. This would also enable researchers to investigate the specificity of attentional bias in aggression. The results reported here suggest that an attentional bias for angry faces is characterized by relatively undifferentiated ERPs. However, it is not clear whether these findings reflect a general negative attentional bias or whether this ERP pattern is distinct for attentional bias to aggressive stimuli.

To overcome the limitation of the cross-sectional design, and to explore the causal role of cognitive biases in aggression, we suggest either using longitudinal designs, or implementing emotion induction between two assessments of attentional bias. 
Attention interacts with a number of other processes. In particular, White, Suway, Pine, Bar-Haim, and Fox (2011) highlight the need to investigate attentional bias along with interpretation bias. They found that preferential allocation of attention affected how ambiguous information was interpreted. Bowler et al. (2017) also used CBM techniques in anxious individuals to investigate whether implementing positive interpretation or attention training also had positive effects on the untrained cognitive domain. They found that attentional bias training resulted in a reduced threat-related attentional bias and an increase in positive interpretation bias. In contrast, some authors have proposed that hostile interpretation bias may not come from an attention bias but from a predisposition to automatically interpret ambiguous situations in a hostile manner (Horsley et al., 2010; Wilkowski \& Robinson, 2008, 2010). Taken together, these results demonstrate the need for further work investigating the cognitive mechanisms which underlie both attention and interpretation processes. For instance, Gagnon and colleagues have assessed the neural correlates of interpretation bias in aggression (Gagnon et al., 2016; Gagnon et al., 2017). They found that across both aggressive and non-aggressive samples, during a sentence completion task, participants showed increased N400 amplitude in response to non-hostile target words that violated the expectations of hostile scenarios. Within the aggression literature, it will be important to consider whether similar neural processes contribute to biases at both the attention and interpretation phases of processing.

\subsection{Conclusion}

Increasing physical aggression scores were associated with faster reaction times to probes replacing angry relative to neutral faces, but there was no differentiation in P3 amplitudes across trial types among participants scoring more highly on the physical aggression. In contrast, individuals with low aggression scores exhibited increased amplitude to anger-congruent trials at right parietal sites. The similarity of P3 amplitude across angry and neutral trials and increased susceptibility to selective visual processing in high physical aggression individuals suggests processing abnormalities in valence-driven attentional selectivity among this population. Physical aggression 
was correlated with enhanced amplitude to anger-incongruent relative to anger-congruent probes. We suggest that individuals with high physical aggression recruit greater cognitive resources in inhibiting the response to angry face distractors on these neutral trials. These findings shed new light on the cognitive foundations of aggression, and could inform the development of novel therapeutic strategies for modifying visual attentional bias in physical aggression.

\section{Methods and Materials}

\subsection{Participants}

Data were collected from 36 university student and staff volunteers. To take part in the study participants had to be male, aged between 18 and 35, speak English as their first language (due to the study being part of a larger programme of research that included text-based attentional and interpretive bias tests) and have normal or corrected vision. Exclusion criteria were: diagnosed with a psychological condition in the last 12 months; receiving psychological treatment; or taken anabolic steroids within the past three months.

One participant whose first language was not English was excluded. Two further participants were excluded from analysis due to excessive noise during EEG recording. This resulted in a final sample of 33 for all continuous analyses. Two participants had scores that equalled the median and consequently could not be categorized, therefore both behavioural and ERP between-subjects analyses included a sample of 31 participants (16 high physical aggression, 15 low physical aggression).

Efforts were made to recruit participants with a wide range of aggression scores by, for example, distributing adverts that included questions such as 'Do you tend to lose your temper?' and 'Do you frequently get road rage?' Participants varied in age from 18 to $35(M=21.77, S D=$ 4.55). All participants had completed schooling to 18 years of age, and $20 \%$ had completed an undergraduate or higher university degree. 


\subsection{Self-report measures}

\subsubsection{Aggression Questionnaire (BPAQ; Buss \& Perry, 1992).}

The BPAQ consists of 29 statements on a 5-point Likert scale, which ranges from 1 ("Extremely uncharacteristic of me") to 5 ("Extremely characteristic of me"). There are four subscales: nine items measure physical aggression; five verbal aggression; eight anger; and eight hostility. Example physical aggression items include: "If I have to resort to violence to protect my rights, I will" and "Given enough provocation, I may hit another person". Higher summed scores represent a higher level of aggression. The physical aggression scale has good reported internal consistency $(\alpha=.85)$ (Buss \& Perry, 1992).

\subsubsection{Attentional Control Scale (ACS; Derryberry \& Reed, 2002).}

Attentional control was measured because it is thought to contribute to attentional bias, which is conceptualized as the bottom-up stimulus-driven capture of attention combined with suboptimal attentional control (e.g., Wiers et al., 2013). Attentional control is negatively associated with aggression (e.g., Muris, van der Pennen, Sigmond, \& Mayer, 2008). The ACS contains 20 selfrated statements that probe participants' perceived levels of attentional shifting (e.g., "It is easy for me to alternate between two different tasks") and attentional focusing (e.g., "My concentration is good even if there is music in the room around me"). Each item is rated on a 4point scale that ranges from 1 (almost never) to 4 (always). Eleven items are reverse-scored and all items summed. A higher total score represents better attentional control. The ACS has good reported reliability with reported Cronbach's alpha values between .71 (Verwoerd, de Jong, \& Wessel, 2008) and .88 (Derryberry \& Reed, 2001).

\subsubsection{Delinquency Questionnaire (DQ; (Tarry \& Emler, 2007).}

The DQ requires respondents to indicate how many times they have engaged in delinquent behaviour in the past 12 months by rating 24 statements (e.g., "Thrown stones at cars, trains, 
buses or other vehicles") on a scale from 0 (never) to 3 (several times). Scores are summed (range 0 to 72 ), with higher total scores representing more frequent delinquency. The scale has excellent reported reliability $(\alpha=.94$; Tarry $\&$ Emler, 2007).

4.2.4 State-Trait Anxiety Inventory (STAI-T; (Spielberger, Jacobs, Russell, \& Crane, 1983). Trait anxiety has been associated with selective attention to angry faces and hence was assessed as a possible covariate of attentional bias (e.g., Bradley, Mogg, Falla, \& Hamilton, 1998; Byrne \& Eysenck, 1995). The STAI-T comprises 20 items on which participants rate how they generally feel (e.g., "I feel pleasant", "I feel nervous and restless") on a 4-point scale from 1 (almost never) to 4 (almost always) (Spielberger et al., 1983). Positive statements are reverse scored and a composite score is generated by summing the individual items. The scale has good reported reliability and internal consistency with Cronbach's alphas ranging from .86 to .95 (Barnes, Harp, \& Jung, 2002).

\subsection{Experimental task}

\subsubsection{Dot-probe task.}

Attentional bias was measured using the probe classification version of the dot-probe task ${ }^{1}$, adapted from MacLeod, Rutherford, Campbell, Ebsworthy, and Holker (2002), and programmed using E-Prime software (Schneider, Eschman, \& Zuccolotto, 2002). Stimuli consisted of 12 angry and 12 neutral facial expressions. These were colour images obtained from the Chicago Face Database (Ma, Correll, \& Wittenbrink, 2015). Individual images were cropped to dimensions of $7.9 \mathrm{~cm}$ by $11.9 \mathrm{~cm}$ and resized to $50 \%$ of originals in Photoshop, such that each face was just under $4 \mathrm{~cm}$ by $6 \mathrm{~cm}$ onscreen. All images portrayed White male actors (12 in total; the same actor displayed the angry and neutral facial expressions in each pair) against a white background.

\footnotetext{
${ }^{1}$ Participants also completed a second dot-probe task which was identical to that outlined above although stimuli were replaced with threat-related and neutral words instead of faces (in preparation).
} 
Participants were seated $60 \mathrm{~cm}$ from a 23 -inch monitor (black text or colour images on a white background), affording a visual angle of approximately 3 degrees between items (cf. See, MacLeod, \& Bridle, 2009). A set of 12 face pairs was presented for 500ms in randomized order, separated by a vertical distance of $3 \mathrm{~cm}$ above and below the central fixation cross. There were a total of 96 trials, with each of the 12 face pairs being presented eight times. Each trial began with a fixation point (three small crosses) in the centre of the computer screen for varying duration (range 1060 to $1973 \mathrm{~ms}$ ), followed by presentation of the stimulus pair. Next, a left- or rightpointing arrow probe (“<” vs. " >”) appeared in the prior location of the angry or neutral stimulus until response (see Figure 4). The direction (left or right) and location (top or bottom) of the arrow probe was equally distributed across trial types and presentation order was randomized throughout the test. Participants were instructed to identify the direction of the on screen arrow probe using the arrow keys as quickly and accurately as possible. A 1-second blink screen followed the target response to minimize ERP artefacts, after which the next trial started immediately. Hostile attentional bias is characterized by faster reaction times to arrow probes that appear in the location of previously presented angry faces (congruent trials) compared with probes located in the place of previously presented neutral faces (incongruent trials). There were 10 practice trials and a break occurred halfway through the test. 


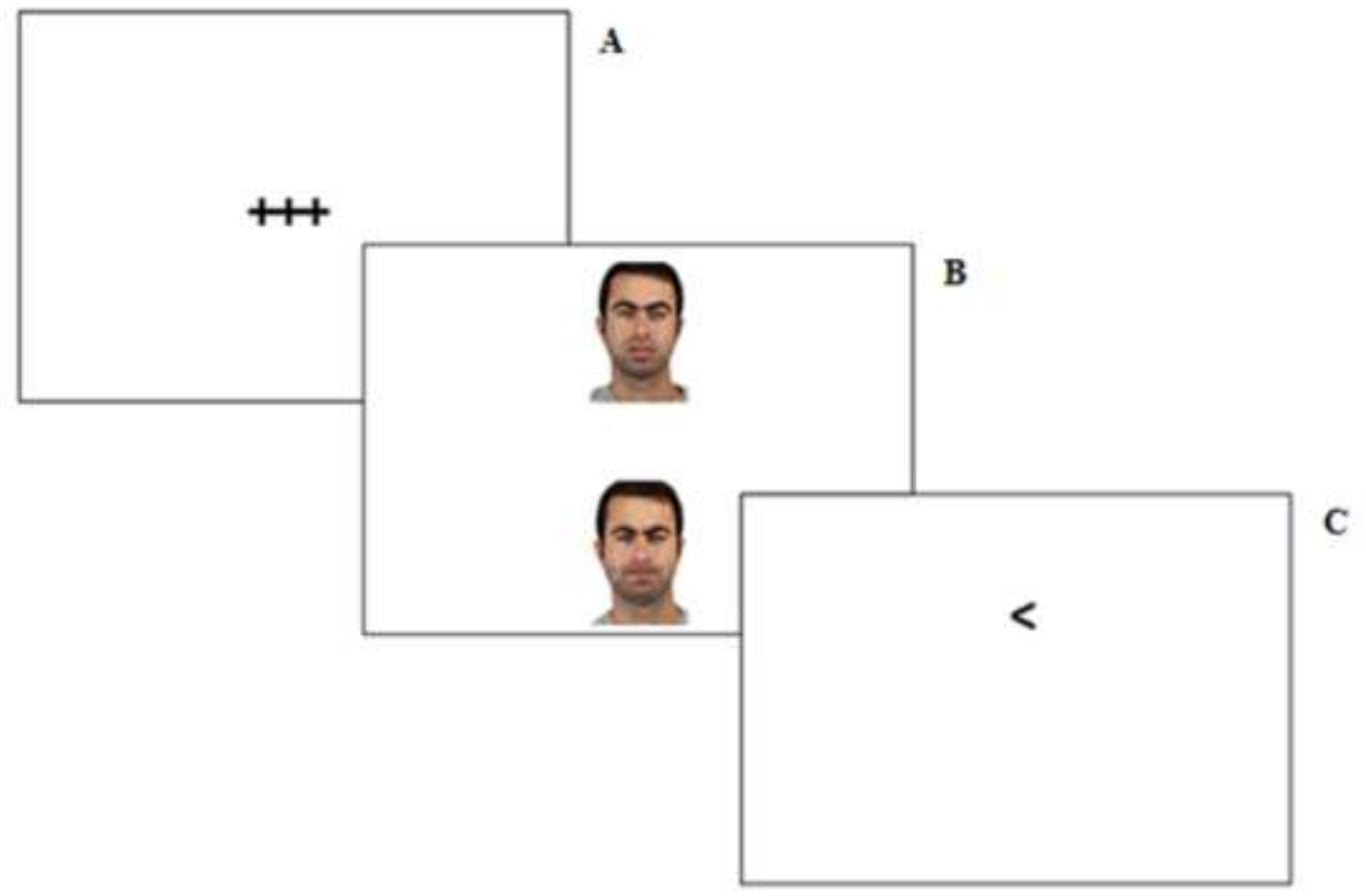

Figure 4. Procedure for the dot-probe task; a) fixation cross is presented in the centre of the screen for a randomized time between 1060 and $1973 \mathrm{~ms}$; b) the face pair is presented for 500ms; c) an arrow probe is presented in the prior location of either the angry or neutral face and stays on screen until participant response (pressing either the left or right arrow key).

\subsubsection{EEG acquisition}

EEG was recorded with a 32-channel active electrode system (Brain Products $\mathrm{GmbH}$ ) embedded in a nylon cap (10/10 system extended). An additional electrode was placed under the left eye to monitor vertical eye movements (lower EOG). The continuous EEG signal was acquired at a 500 $\mathrm{Hz}$ sampling rate using $\mathrm{FCz}$ as reference. The impedance was measured before EEG recording and was below $20 \mathrm{k} \Omega$.

\subsection{Procedure}

Initially participants were asked to complete an online questionnaire (Qualtrics software); this involved giving consent, reporting demographic information and completing the BPAQ. During the laboratory session, which took place up to one month after the online questionnaire, 
participants read and signed consent forms. They were then fitted with a nylon cap embedded with 32 electrodes and EEG was recorded during completion of the dot-probe task. The ERP pictorial attentional bias test was the second procedure of three computerized tasks that participants completed during the laboratory session (participants also completed a further dotprobe task containing angry and neutral words followed by a recognition task as a measure of interpretation bias, results of which are reported elsewhere, paper in preparation). Participants also completed paper copies of the four questionnaires (ACS, DQ, STAI-T and the Ambiguous Intentions Hostility Questionnaire: AIHQ; Combs, Penn, Wicher, \& Waldheter, 2007). To minimize order effects, completion of the computer-based tasks and questionnaires was counterbalanced. The testing session took approximately 90 minutes and participants received course credits or shopping vouchers (£10) as compensation.

\subsection{Data analysis}

\subsubsection{Questionnaires.}

The DQ and STAI-T had no missing items. The ACS and the BPAQ (physical aggression subscale) each had one case of missing data. Missing values were replaced with the mean of the completed items for each questionnaire (method used by Judah, Grant, Mills, \& Lechner, 2014). This simple approach was selected as it is considered to make relatively little difference if missing data represent less than 5\% of the dataset (Tabachnick \& Fidell, 2013).

The BPAQ $(\alpha=.92)$, Physical Aggression subscale from BPAQ $(\alpha=.90)$, DQ $(\alpha=.81)$, and STAI-T $(\alpha=.94)$ demonstrated good internal reliability. The ACS was only moderately reliable $(\alpha=.66)$. All questionnaire and attentional bias scores were assessed for normality; skewness and kurtosis were divided by their corresponding standard error. All resultant values were between the recommended ranges of \pm 2 (Field, 2013), and therefore parametric analyses were conducted. 
Anxiety did not correlate with physical aggression, $r(31)=.080, p=.660$, or attentional bias, $r(31)=-.159, p=.378$, and was therefore not included as a covariate in the main analyses. Attentional control did not correlate with physical aggression, $r(31)=.079, p=.663$, or attentional bias, $r(31)=-.035, p=.845$, and was therefore also excluded. Physical aggression correlated with delinquency, $r(31)=.403, p=.020$. However, delinquency data were not considered further due to a floor effect in our non-clinical sample $(M d n=3.0$, range $=0-21)$.

\subsubsection{Behavioural data.}

Median reaction times to probes replacing angry and neutral faces were extracted as they were not skewed by extreme scores (e.g., Whelan, 2008). Reaction times were evaluated on correct trials only (3372 out of $3456(97.6 \%))$. An attentional bias index was calculated by subtracting the median reaction time on neutral trials from the median reaction time on angry trials. Therefore, a negative bias score indicates that participants responded more rapidly when probes replaced angry than neutral faces.

We used both median-split and correlational approaches to evaluate how levels of physical aggression in participants were related to attentional bias. Participants were categorized as high aggression or low aggression according to their score on the physical aggression subscale.

Reaction times to probes replacing angry and neutral faces, and the derived attentional bias index, were normally distributed. Pearson's $r$ (2-tailed) was used to assess whether physical aggression significantly correlated with attentional bias. A one-way ANOVA was conducted to assess if there was a significant effect of congruency (probe replaced angry face versus neutral face) between high and low physical aggression groups. Paired-samples t-tests were used to compare reaction times to angry and neutral faces within each group, and independent-samples ttests were used to compare reaction times between groups (see Table 1).

\subsubsection{EEG data.}


Offline analyses were conducted using EEGLAB (Delorme \& Makeig, 2004) and ERPLAB (Lopez-Calderon \& Luck, 2014), which are open source toolboxes running under Matlab 7.12 (R2013a, The Mathworks). The EEG data was not re-referenced offline. High- and low-pass filter half-amplitude cut-offs were set at 0.1 and $40 \mathrm{~Hz}$, respectively. Before averaging, trials contaminated by excessive artifacts such as eye blinks were rejected automatically using a step function (Luck, 2005) with a voltage threshold of $\pm 100 \mu \mathrm{V}$ in moving windows of $200 \mathrm{~ms}$ and with a window step of $100 \mathrm{~ms}$. Noisy channels were interpolated using the EEGLAB function eeg_interp (spherical interpolation).

The EEG was segmented into epochs of $1200 \mathrm{~ms}$; from $-200 \mathrm{~ms}$ to $1000 \mathrm{~ms}$ post stimulus (face) onset. A pre-face baseline was chosen to avoid possible introduction of post-probe condition differences created by a mid-trial change in baseline (Mingtian et al., 2011; Poulsen et al., 2005), a potential confound which was confirmed in our ERP analyses (see above). Mean amplitude between $300 \mathrm{~ms}$ and $500 \mathrm{~ms}$ post arrow probe (corresponding to $800-1000 \mathrm{~ms}$ post-face onset) onset was extracted for statistical analyses of the probe-evoked P3 effect. ERPs were timelocked to the onset of each face pair. Analysis focused on posterior parietal electrode sites, including $\mathrm{CP} 1 / 2, \mathrm{P} 7 / 8, \mathrm{P} 3 / \mathrm{P} 4$ and TP9/10, where $\mathrm{P} 3$ component is considered to be maximal (e.g., Polich, 2007).

A mixed model analysis of variance (ANOVA) was performed on post-probe ERP measures for the region of interest, with the following within-subjects factors: congruency (probe replaced angry versus neutral); electrode (4 levels); and hemisphere (left versus right). Physical aggression group was added as the between-subjects factor. Greenhouse-Geisser $F$-tests (Geisser $\&$ Greenhouse, 1958) are reported throughout for all repeated measures to avoid violations of the sphericity assumption.

For consistency between behavioural and ERP data analyses, Pearson's correlations (2tailed) were also calculated to investigate the relationship between mean P3 amplitude to angry relative to neutral face stimuli and physical aggression scores. 


\section{Acknowledgments}

Funding sources: This research was supported by a studentship from the University of East Anglia awarded to the first author. It did not receive any grant from funding agencies in the public, commercial, or not-for-profit sectors. Conflicts of interest disclosure: There are no conflicts of interest arising from the research reported in this article.

\section{References}

Archer, J. (2004). Sex differences in aggression in real-world settings: A meta-analytic review. Review of General Psychology, 8(4), 291-322. doi: 10.1037/1089-2680.8.4.291

Bar-Haim, Y. (2010). Research review: attention bias modification (ABM): A novel treatment for anxiety disorders. Journal of Child Psychology and Psychiatry, 51(8), 859-870. doi: $10.1111 / \mathrm{j} .1469-7610.2010 .02251 . \mathrm{x}$

Bar-Haim, Y., Lamy, D., Pergamin, L., Bakermans-Kranenburg, M. J., \& Van Ijzendoorn, M. H. (2007). Threat-related attentional bias in anxious and nonanxious individuals: A metaanalytic study. Psychological Bulletin, 133(1), 1-24. http://dx.doi.org/10.1037/0033$\underline{2909.133 .1 .1}$

Barnes, L. L., Harp, D., \& Jung, W. S. (2002). Reliability generalization of scores on the Spielberger state-trait anxiety inventory. Educational and Psychological Measurement, 62(4), 603-618. https://doi.org/10.1177/0013164402062004005

Beck, A. T., \& Clark, D. A. (1997). An information processing model of anxiety: Automatic and strategic processes. Behaviour Research and Therapy, 35(1), 49-58. https://doi.org/10.1016/S0005-7967(96)00069-1

Bernat, E. M., Hall, J. R., Steffen, B. V., \& Patrick, C. J. (2007). Violent offending predicts P300 amplitude. International Journal of Psychophysiology, 66(2), 161-167. https://doi.org/10.1016/j.ijpsycho.2007.03.021 
Bertsch, K., Böhnke, R., Kruk, M. R., \& Naumann, E. (2009). Influence of aggression on information processing in the emotional Stroop task-an event-related potential study. Frontiers in Behavioral Neuroscience, 3(28), 1-10. https://doi.org/10.3389/neuro.08.028.2009

Bishop, S. J. (2008). Neural mechanisms underlying selective attention to threat. Annals of the New York Academy of Sciences, 1129(1), 141-152. doi: 10.1196/annals.1417.016

Bowler, J., Hoppitt, L., Illingworth, J., Dalgleish, T., Ononaiye, M., Perez-Olivas, G., \& Mackintosh, B. (2017). Asymmetrical transfer effects of cognitive bias modification: Modifying attention to threat influences interpretation of emotional ambiguity, but not vice versa. Journal of Behavior Therapy and Experimental Psychiatry, 54, 239-246. https://doi.org/10.1016/j.jbtep.2016.08.011

Bradley, B. P., Mogg, K., Falla, S. J., \& Hamilton, L. R. (1998). Attentional bias for threatening facial expressions in anxiety: Manipulation of stimulus duration. Cognition \& Emotion, 12(6), 737-753. http://dx.doi.org/10.1080/026999398379411

Brugman, S., Lobbestael, J., von Borries, A. K. L., Bulten, B. E. H., Cima, M., Schuhmann, T., ... \& Arntz, A. (2016). Cognitive predictors of violent incidents in forensic psychiatric inpatients. Psychiatry Research, 237, 229-237. https://doi.org/10.1016/i.psychres.2016.01.035

Buss, A. H., \& Perry, M. (1992). The aggression questionnaire. Journal of Personality and Social Psychology, 63(3), 452-459. http://dx.doi.org/10.1037/0022-3514.63.3.452

Byrne, A., \& Eysenck, M. W. (1995). Trait anxiety, anxious mood, and threat detection. Cognition \& Emotion, 9(6), 549-562. http://dx.doi.org/10.1080/02699939508408982 Clarke, P. J., MacLeod, C., \& Guastella, A. J. (2013). Assessing the role of spatial engagement and disengagement of attention in anxiety-linked attentional bias: a critique of current paradigms and suggestions for future research directions. Anxiety, Stress \& Coping, 26(1), 1-19. doi: $10.1080 / 10615806.2011 .638054$ 
Coccaro, E. F., McCloskey, M. S., Fitzgerald, D. A., \& Phan, K. L. (2007). Amygdala and orbitofrontal reactivity to social threat in individuals with impulsive aggression. Biological Psychiatry, 62(2), 168-178. doi: 10.1016/j.biopsych.2006.08.024

Coles, M. G. H., Smid, H. G. O. M, Scheffers, M. K., \& Otten, L. J. (1995). Mental chronometry and the study of human information processing. In Rugg, M. D., \& Coles, M. G. H. (Eds.), Electrophysiology of mind: Event-related brain potentials and cognition (pp. 86131). New York: Oxford University Press.

doi: 10.1093/acprof:oso/9780198524168.003.0004

Combs, D. R., Penn, D. L., Wicher, M., \& Waldheter, E. (2007). The Ambiguous Intentions Hostility Questionnaire (AIHQ): a new measure for evaluating hostile social-cognitive biases in paranoia. Cognitive Neuropsychiatry, 12(2), 128-143.

Crick, N. R., \& Dodge, K. A. (1994). A review and reformulation of social informationprocessing mechanisms in children's social adjustment. Psychological Bulletin, 115(1), 74-101. http://dx.doi.org/10.1037/0033-2909.115.1.74

Davidson, R. J. (1993). Cerebral asymmetry and emotion: Conceptual and methodological conundrums. Cognition \& Emotion, 7(1), 115-138.

Dehghani, M., Sharpe, L., Nicholas, M. (2003). Selective attention to pain-related information is chronic musculo-skeletal pain patients. Pain, 105, 37-46. https://doi.org/10.1016/S0304$\underline{3959(03) 00224-0}$

Delorme, A., \& Makeig, S. (2004). EEGLAB: an open source toolbox for analysis of single-trial EEG dynamics including independent component analysis. Journal of Neuroscience Methods, 134(1), 9-21. doi: 10.1016/j.jneumeth.2003.10.009

Derryberry, D., \& Reed, M. A. (2001). 13 A multidisciplinary perspective on attentional control. In Folk, C., \& Gibson, B (Eds.), Attention, distraction, and action: Multiple perspectives on attentional capture (pp. 325-347). London: Elsevier Science. 
Derryberry, D., \& Reed, M. A. (2002). Anxiety-related attentional biases and their regulation by attentional control. Journal of Abnormal Psychology, 111(2), 225-236. http://dx.doi.org/10.1037/0021-843X.111.2.225

Desimone, R., \& Duncan, J. (1995). Neural mechanisms of selective visual attention. Annual Review of Neuroscience, 18(1), 193-222. doi: 10.1146/annurev.ne.18.030195.001205

Field, A. (2013). Discovering statistics using IBM SPSS statistics. London: Sage.

Gagnon, J., Aubin, M., Emond, F. C., Derguy, S., Bessette, M., \& Jolicoeur, P. (2016). Neural mechanisms underlying attribution of hostile intention in nonaggressive individuals: An ERP study. International Journal of Psychophysiology, 110, 153-162.

\section{https://doi.org/10.1016/j.ijpsycho.2016.08.007}

Gagnon, J., Aubin, M., Emond, F. C., Derguy, S., Brochu, A. F., Bessette, M., \& Jolicoeur, P. (2017). An ERP study on hostile attribution bias in aggressive and nonaggressive individuals. Aggressive Behavior, 43(3), 217-229. https://doi.org/10.1002/ab.21676

Gao, Y., Raine, A., Venables, P. H., \& Mednick, S. A. (2013). The association between P3 amplitude at age 11 and criminal offending at age 23. Journal of Clinical Child \& Adolescent Psychology, 42(1), 120-130. http://dx.doi.org/10.1080/15374416.2012.719458

Geisser, S., \& Greenhouse, S. W. (1958). An extension of Box's results on the use of the F distribution in multivariate analysis. The Annals of Mathematical Statistics, 29(3), 885891. doi:10.1214/aoms/1177706545

Helfritz-Sinville, L. E., \& Stanford, M. S. (2015). Looking for trouble? Processing of physical and social threat words in impulsive and premeditated aggression. The Psychological Record, 65(2), 301-314. https://doi.org/10.1007/s40732-014-0106

Judah, M. R., Grant, D. M., Mills, A. C., \& Lechner, W. V. (2014). Factor structure and validation of the attentional control scale. Cognition \& Emotion, 28(3), 433-451. http://dx.doi.org/10.1080/02699931.2013.835254 
Knight, R. T., Scabini, D., Woods, D. L., \& Clayworth, C. C. (1989). Contributions of temporalparietal junction to the human auditory P3. Brain Research, 502(1), 109-116. https://doi.org/10.1016/0006-8993(89)90466-6

Koster, E. H., Crombez, G., Verschuere, B., \& De Houwer, J. (2004). Selective attention to threat in the dot probe paradigm: Differentiating vigilance and difficulty to disengage. Behaviour Research and Therapy, 42(10), 1183-1192. https://doi.org/10.1016/j.brat.2003.08.001

Leutgeb, V., Sarlo, M., Schöngassner, F., \& Schienle, A. (2015). Out of sight, but still in mind: electrocortical correlates of attentional capture in spider phobia as revealed by a 'dot probe' paradigm. Brain and Cognition, 93, 26-34.

doi: 10.1016/j.bandc.2014.11.005

Lopez-Calderon, J., \& Luck, S. J. (2014). ERPLAB: an open-source toolbox for the analysis of event-related potentials. Frontiers in Human Neuroscience, 8, 1-14. doi: 10.3389/fnhum.2014.00213

Luck, S. J. (2005). An introduction to the event-related potential technique. Cambridge, Mass.: MIT Press.

Ma, D. S., Correll, J., \& Wittenbrink, B. (2015). The Chicago face database: A free stimulus set of faces and norming data. Behavior Research Methods, 47(4), 1122-1135. doi: 10.3758/s13428-014-0532-5

MacLeod, C., Mathews, A., \& Tata, P. (1986). Attentional bias in emotional disorders. Journal of Abnormal Psychology, 95(1), 15-20. http://dx.doi.org/10.1037/0021-843X.95.1.15

MacLeod, C., Rutherford, E., Campbell, L., Ebsworthy, G., \& Holker, L. (2002). Selective attention and emotional vulnerability: assessing the causal basis of their association through the experimental manipulation of attentional bias. Journal of Abnormal Psychology, 111(1), 107-123. http://dx.doi.org/10.1037/0021-843X.111.1.107 
MacNamara, A., Kappenman, E. S., Black, S. R., Bress, J. N., \& Hajcak, G. (2013). Integrating behavioral and electrocortical measures of attentional bias toward threat. In: K. C. Barrett, N. A. Fox, G. A. Morgan, D. J. Fidler, \& L. A. Daunhauer (Eds.), Handbook of selfregulatory processes in development: New directions and international perspectives (pp. 215-242). New York: Psychology Press. doi: 10.4324/9780203080719.ch11

Maoz, K., Adler, A. B., Bliese, P. D., Sipos, M. L., Quartana, P. J., \& Bar-Haim, Y. (2017). Attention and interpretation processes and trait anger experience, expression, and control. Cognition and Emotion, 1-12. http://dx.doi.org/10.1080/02699931.2016.1231663

MatLab 7.12. [Computer software]. Natick, MA: The MathWorks, Inc.

Mellentin, A. I., Dervisevic, A., Stenager, E., Pilegaard, M., \& Kirk, U. (2015). Seeing enemies? A systematic review of anger bias in the perception of facial expressions among angerprone and aggressive populations. Aggression and Violent Behavior, 25, 373-383. https://doi.org/10.1016/j.avb.2015.09.001

Mingtian, Z., Xiongzhao, Z., Jinyao, Y., Shuqiao, Y., \& Atchley, R. A. (2011). Do the early attentional components of ERPs reflect attentional bias in depression? It depends on the stimulus presentation time. Clinical Neurophysiology, 122(7), 1371-1381. doi: 10.1016/j.clinph.2010.09.016.

Mogg, K., Bradley, B. P., Millar, N., \& White, J. (1995a). A follow-up study of cognitive bias in generalized anxiety disorder. Behaviour Research and Therapy, 33(8), 927-935. https://doi.org/10.1016/0005-7967(95)00031-R

Mogg, K., Bradley, B. P., Williams, R., \& Mathews, A. (1993). Subliminal processing of emotional information in anxiety and depression. Journal of Abnormal Psychology, 102, 304-311. http://dx.doi.org/10.1037/0021-843X.102.2.304

Mogg, K., Bradley, B. P., \& Williams, R. (1995b). Attentional bias in anxiety and depression: the role of awareness. British Journal of Clinical Psychology, 34, 17-36. doi: 10.1111/j.2044-8260.1995.tb01434.x 
Mogg, K., \& Bradley, B. P. (1998). A cognitive-motivational analysis of anxiety. Behaviour Research and Therapy, 36(9), 809-848. https://doi.org/10.1016/S0005-7967(98)00063-1

Mogg, K., \& Bradley, B. P. (2002). Selective orienting of attention to masked threat faces in social anxiety. Behaviour Research and Therapy, 40(12), 1403-1414.

Mogoaşe, C., David, D., \& Koster, E. H. (2014). Clinical efficacy of attentional bias modification procedures: An updated metaanalysis. Journal of Clinical Psychology, 70(12), 1133-1157. doi: $10.1002 /$ jclp.22081

Muris, P., van der Pennen, E., Sigmond, R., \& Mayer, B. (2008). Symptoms of anxiety, depression, and aggression in non-clinical children: Relationships with self-report and performance-based measures of attention and effortful control. Child Psychiatry and Human Development, 39(4), 455-467. doi: 10.1007/s10578-008-0101-1

Palermo, R., \& Rhodes, G. (2007). Are you always on my mind? A review of how face perception and attention interact. Neuropsychologia, 45(1), 75-92. doi: $10.1016 /$ j.neuropsychologia.2006.04.025

Patrick, C. J. (2008). Psychophysiological correlates of aggression and violence: An integrative review. Philosophical Transactions of the Royal Society B: Biological Sciences, 363(1503), 2543-2555. doi: 10.1098/rstb.2008.0028

Polich, J. (2007). Updating P300: An integrative theory of P3a and P3b. Clinical Neurophysiology, 118(10), 2128-2148. doi: 10.1016/j.clinph.2007.04.019

Posner, M. I. (1980). Orienting of attention. Quarterly Journal of Experimental Psychology, 32(1), 3-25. https://doi.org/10.1080/00335558008248231

Poulsen, C., Luu, P., Davey, C., \& Tucker, D. M. (2005). Dynamics of task sets: evidence from dense-array event-related potentials. Cognitive Brain Research, 24(1), 133-154. https://doi.org/10.1016/j.cogbrainres.2005.01.008 
Pourtois, G., Grandjean, D., Sander, D., \& Vuilleumier, P. (2004). Electrophysiological correlates of rapid spatial orienting towards fearful faces. Cerebral Cortex, 14(6), 619-633. https://doi.org/10.1093/cercor/bhh023

Sass, S. M., Evans, T. C., Xiong, K., Mirghassemi, F., \& Tran, H. (2017). Attention training to pleasant stimuli in anxiety. Biological Psychology, 122, 80-92. https://doi.org/10.1016/j.biopsycho.2016.03.003

Schneider, W., Eschman, A., \& Zuccolotto, A. (2002). E-Prime: User's guide: Psychology Software Incorporated.

See, J., MacLeod, C., \& Bridle, R. (2009). The reduction of anxiety vulnerability through the modification of attentional bias: a real-world study using a home-based cognitive bias modification procedure. Journal of Abnormal Psychology, 118(1), 65.

doi: $10.1037 / \mathrm{a} 0014377$

Shulman, G. L., Pope, D. L., Astafiev, S. V., McAvoy, M. P., Snyder, A. Z., \& Corbetta, M. (2010). Right hemisphere dominance during spatial selective attention and target detection occurs outside the dorsal frontoparietal network. Journal of Neuroscience, 30(10), 36403651. https://doi.org/10.1523/JNEUROSCI.4085-09.2010

Smith, P., \& Waterman, M. (2003). Processing bias for aggression words in forensic and nonforensic samples. Cognition \& Emotion, 17(5), 681-701. http://dx.doi.org/10.1080/02699930302281

Spielberger, C. D., Jacobs, G. A., Russell, S., \& Crane, R. S. (1983). Assessment of anger: The state-trait anger scale. In J. N. Butcher \& C. D. Spielberger (Eds.), Advances in personality assessment (Vol. 2, pp. 52-76). Hillsdale, NJ: Erlbaum.

Strack, F., \& Deutsch, R. (2004). Reflective and impulsive determinants of social behavior. Personality and Social Psychology Review, 8(3), 220-247. doi: $10.1207 /$ s15327957pspr0803_1 
Surguy, S. M., \& Bond, A. J. (2006). P300 to emotionally relevant stimuli as an indicator of aggression levels. Aggressive Behavior, 32(3), 253-260. doi: 10.1002/ab.20124

Qualtrics software, Version [October, 2015]. Copyright C [2017] Qualtrics. Qualtrics and all other Qualtrics product or service names are registered trademarks or trademarks of Qualtrics, Provo, UT, USA. http://www.qualtrics.com

Tabachnick, B. G., \& Fidell, L. S. (2013). Using multivariate statistics: Pearson Education.

Tarry, H., \& Emler, N. (2007). Attitudes, values and moral reasoning as predictors of delinquency. British Journal of Developmental Psychology, 25(2), 169-183. doi: 10.1348/026151006X113671

van Dinteren, R., Arns, M., Jongsma, M. L., \& Kessels, R. P. (2014). P300 development across the lifespan: A systematic review and meta-analysis. PLoS ONE, 9(2), e87347

Verleger, R., Heide, W., Butt, C., \& Kömpf, D. (1994). Reduction of P3 b in patients with temporo-parietal lesions. Cognitive Brain Research, 2(2), 103-116. https://doi.org/10.1016/0926-6410(94)90007-8

Verwoerd, J., de Jong, P. J., \& Wessel, I. (2008). Low attentional control and the development of intrusive memories following a laboratory stressor. Journal of Psychopathology and Behavioral Assessment, 30(4), 291-297. https://doi.org/10.1007/s10862-008-9080-6

Whelan, R. (2008). Effective analysis of reaction time data. The Psychological Record, 58(3), 475. https://doi.org/10.1007/BF03395630

White, L. K., Suway, J. G., Pine, D. S., Bar-Haim, Y., \& Fox, N. A. (2011). Cascading effects: The influence of attention bias to threat on the interpretation of ambiguous information. Behaviour Research and Therapy, 49(4), 244-251. https://doi.org/10.1016/j.brat.2011.01.004

Wiers, R. W., Gladwin, T. E., Hofmann, W., Salemink, E., \& Ridderinkhof, K. R. (2013). Cognitive bias modification and cognitive control training in addiction and related 
psychopathology mechanisms, clinical perspectives, and ways forward. Clinical Psychological Science, 1(2), 192-212. https://doi.org/10.1177/2167702612466547

Wilkowski, B. M., \& Robinson, M. D. (2010). The anatomy of anger: An integrative cognitive model of trait anger and reactive aggression. Journal of Personality, 78(1), 9-38. doi: 10.1111/j.1467-6494.2009.00607.x

Yamaguchi, S., \& Knight, R. T. (1992). Effects of temporal-parietal lesions on the somatosensory P3 to lower limb stimulation. Electroencephalography and Clinical Neurophysiology/Evoked Potentials Section, 84(2), 139-148.

https://doi.org/10.1016/0168-5597(92)90018-7 